\title{
THE SIMPLEST STATIONARY SUBALGEBRAS, FOR COMPACT LINEAR LIE ALGEBRAS
}

\author{
O. G. STYRT
}

\begin{abstract}
Sufficient conditions are obtained for the existence of a vector with a one-dimensional or simple three-dimensional stationary subalgebra for an irreducible compact linear Lie algebra.
\end{abstract}

\section{$\S 1$. INTRODUCTION}

Let $V_{\mathbb{R}}$ be a real vector space, and $\mathfrak{g}_{\mathbb{R}} \subset \mathfrak{s l}\left(V_{\mathbb{R}}\right)$ an irreducible compact linear Lie algebra of rank $r>1$ that has simple derived subalgebra.

We are interested in the question of whether there exists a vector $v \in V_{\mathbb{R}}$ whose stationary subalgebra is isomorphic to one of the algebras $\mathbb{R}$ or $\mathfrak{s u}_{2}$, that is, whether

$$
\exists v \in V_{\mathbb{R}}: \operatorname{rk}\left(\left(\mathfrak{g}_{\mathbb{R}}\right)_{v}\right)=1 .
$$

This problem is auxiliary to the problem of finding all compact linear groups for which the topological quotient of the action is homeomorphic to a cell. So far, the cases of a group with commutative connected component [1] and of the simple threedimensional group [2, 3] have been considered. As for an arbitrary linear representation that has a vector with stabilizer of rank 1, we plan to reduce it to the corresponding slice-representation by using the slice theorem [4, Chapter II, $\S \S 4-5]$.

We shall be using the following notation related to representations of complex reductive Lie algebras. The representation of an arbitrary complex reductive Lie algebra dual to a representation $R$ is denoted by $R^{\prime}$. When denoting a direct sum of representations for convenience we shall use the sign " + " instead of the sign " $\oplus$ ".

Let $V$ denote the complex space $V_{\mathbb{R}}(\mathbb{C})$, and $\mathfrak{g}$ the complex reductive linear Lie algebra $\mathfrak{g}_{\mathbb{R}}(\mathbb{C}) \subset\left(\mathfrak{s l}\left(V_{\mathbb{R}}\right)\right)(\mathbb{C})=\mathfrak{s l}(V)$, which by assumption has a simple derived subalgebra. For some faithful irreducible representation $R$ of the Lie algebra $\mathfrak{g}$, the tautological representation $\mathfrak{g}: V$ coincides with one of the representations $R$ or $R+R^{\prime}$. Thus, we can speak about the irreducible linear Lie algebra $R(\mathfrak{g})$ isomorphic to the Lie algebra $\mathfrak{g}$ as an abstract algebra, and about the irreducible simple linear Lie algebra $R([\mathfrak{g}, \mathfrak{g}])$.

If the linear Lie algebra $\mathfrak{g} \subset \mathfrak{s l}(V)$ is reducible, then the space $V_{\mathbb{R}}$ has an $\mathfrak{g}_{\mathbb{R}}$-invariant complex structure, and as a result the complex linear Lie algebra $\mathfrak{g}=\mathfrak{g}_{\mathbb{R}}(\mathbb{C})=\mathfrak{g}_{\mathbb{R}} \oplus i \mathfrak{g}_{\mathbb{R}} \subset$ $\mathfrak{g l}_{\mathbb{C}}\left(V_{\mathbb{R}}\right)$ appears naturally.

When speaking about indecomposable simple root systems, we shall use the standard numbering of simple roots (see, for example, [5], Table 1 or [6], Table 1) and denote the $i$ th basis weight by $\varphi_{i}$.

The aim of this paper is to prove Theorems 1.11 .3

2010 Mathematics Subject Classification. Primary 17B10; Secondary 17B20, 17B45, 22E46.

Key words and phrases. Compact linear Lie algebra, root system, Dynkin diagram, stationary subalgebra in general position (s.s.g.p.)

This research was supported by the "Dinastiya" foundation. 
Theorem 1.1. Suppose that condition (1.1) does not hold, and the (simple) linear Lie algebra $R([\mathfrak{g}, \mathfrak{g}])$ is neither adjoint nor classical.

1. If the linear algebra $\mathfrak{g} \subset \mathfrak{s l}(V)$ is irreducible, then it coincides with one of the following linear algebras:

1) $\varphi_{4}\left(A_{7}\right),\left(\varphi_{1}+\varphi_{2}+\varphi_{3}\right)\left(A_{3}\right)$

2) $\varphi_{r}\left(B_{r}\right),\left(\varphi_{1}+\varphi_{r}\right)\left(B_{r}\right),\left(\varphi_{2}+\varphi_{r}\right)\left(B_{r}\right)(r=3,4)$;

3) $\varphi_{3}\left(B_{4}\right),\left(\varphi_{1}+\varphi_{2}+\varphi_{3}\right)\left(B_{3}\right)$;

4) $\varphi_{2}\left(C_{r}\right)(r>2)$;

5) $\left(\varphi_{1}+\varphi_{3}\right)\left(C_{3}\right),\left(\varphi_{1}+\varphi_{2}+\varphi_{3}\right)\left(C_{3}\right), \varphi_{4}\left(C_{4}\right),\left(\varphi_{2}+\varphi_{4}\right)\left(C_{4}\right), \varphi_{4}\left(C_{5}\right)$;

6) $\varphi_{r}\left(D_{r}\right)(r=5,8)$;

7) $\left(\varphi_{1}+\varphi_{r}\right)\left(D_{r}\right)(r=4,5,8)$;

8) $\varphi_{2}\left(E_{r}\right)(r=7,8)$;

9) $\left(\varphi_{1}+\varphi_{r-1}\right)\left(E_{r}\right)(r=6,8)$;

10) $\varphi_{7}\left(E_{8}\right)$

11) $\varphi_{1}\left(F_{4}\right), \varphi_{2}\left(F_{4}\right), \varphi_{3}\left(F_{4}\right),\left(\varphi_{1}+\varphi_{3}\right)\left(F_{4}\right),\left(\varphi_{1}+\varphi_{4}\right)\left(F_{4}\right),\left(\varphi_{2}+\varphi_{4}\right)\left(F_{4}\right)$;

12) $\varphi_{1}\left(G_{2}\right)$.

2. If the linear algebra $\mathfrak{g} \subset \mathfrak{s l}(V)$ is reducible, then the complex linear Lie algebra $\mathfrak{g} \subset \mathfrak{g l}_{\mathbb{C}}\left(V_{\mathbb{R}}\right)$ coincides with one of the following linear algebras:

1) $\varphi_{2}\left(A_{r}\right)(r>3)$

2) $\left(\varphi_{1}+\varphi_{r-1}\right)\left(A_{r}\right)(r>2)$;

3) $\varphi_{3}\left(A_{r}\right)(r=5,6,7)$;

4) $\varphi_{r}\left(B_{r}\right),\left(\varphi_{1}+\varphi_{r}\right)\left(B_{r}\right),\left(\varphi_{2}+\varphi_{r}\right)\left(B_{r}\right)(r=5,6)$;

5) $\varphi_{3}\left(C_{3}\right),\left(\varphi_{2}+\varphi_{3}\right)\left(C_{3}\right), \varphi_{3}\left(C_{4}\right),\left(\varphi_{1}+\varphi_{4}\right)\left(C_{4}\right)$;

6) $\varphi_{r}\left(D_{r}\right),\left(\varphi_{1}+\varphi_{r}\right)\left(D_{r}\right)(r=6,7)$;

7) $\varphi_{1}\left(E_{6}\right), \varphi_{2}\left(E_{6}\right), \varphi_{1}\left(E_{7}\right), \varphi_{7}\left(E_{7}\right),\left(\varphi_{1}+\varphi_{6}\right)\left(E_{7}\right)$;

8) $\varphi_{2}\left(A_{r-1}\right) \oplus \mathbb{C} E(r>3)$;

9) $\varphi_{3}\left(A_{r-1}\right) \oplus \mathbb{C} E(r=6,7)$;

10) $\varphi_{r-1}\left(B_{r-1}\right) \oplus \mathbb{C} E(r=4,5,6)$;

11) $\left(\varphi_{1}+\varphi_{r-1}\right)\left(B_{r-1}\right) \oplus \mathbb{C} E(r=3, \ldots, 6)$;

12) $\varphi_{2}\left(C_{r-1}\right) \oplus \mathbb{C} E(r>3)$;

13) $\varphi_{3}\left(C_{r-1}\right) \oplus \mathbb{C} E(r=4, \ldots, 7)$;

14) $\left(\varphi_{1}+\varphi_{r-1}\right)\left(C_{r-1}\right) \oplus \mathbb{C} E(r=3,4)$;

15) $\varphi_{r-1}\left(D_{r-1}\right) \oplus \mathbb{C} E(r=6,7,8)$;

16) $\varphi_{1}\left(E_{r-1}\right) \oplus \mathbb{C} E(r=7,8)$;

17) $\varphi_{1}\left(F_{4}\right) \oplus \mathbb{C} E, \varphi_{2}\left(F_{4}\right) \oplus \mathbb{C} E,\left(\varphi_{1}+\varphi_{4}\right)\left(F_{4}\right) \oplus \mathbb{C} E$;

18) $\varphi_{1}\left(G_{2}\right) \oplus \mathbb{C} E,\left(\varphi_{1}+\varphi_{2}\right)\left(G_{2}\right) \oplus \mathbb{C} E$.

Remark. If the (simple) linear Lie algebra $R([\mathfrak{g}, \mathfrak{g}])$ is adjoint, then the following cases are possible:

1) the linear algebra $\mathfrak{g}_{\mathbb{R}} \subset \mathfrak{s l}\left(V_{\mathbb{R}}\right)$ is an adjoint simple compact linear Lie algebra;

2) the linear algebra $\mathfrak{g} \subset \mathfrak{s l}(V)$ is reducible, and the complex linear algebra $\mathfrak{g} \subset \mathfrak{g l}_{\mathbb{C}}\left(V_{\mathbb{R}}\right)$ coincides with the linear algebra $\operatorname{ad}([\mathfrak{g}, \mathfrak{g}]) \oplus \mathbb{C} E \subset \mathfrak{g l}([\mathfrak{g}, \mathfrak{g}])$.

Theorem 1.2. If the linear algebra $\mathfrak{g}_{\mathbb{R}} \subset \mathfrak{s l}\left(V_{\mathbb{R}}\right)$ is an adjoint simple compact linear Lie algebra, then condition (1.1) does not hold. If the linear algebra $\mathfrak{g} \subset \mathfrak{s l}(V)$ is reducible, and the linear algebra $\mathfrak{g} \subset \mathfrak{g l}_{\mathbb{C}}\left(V_{\mathbb{R}}\right)$ coincides with the linear algebra $\operatorname{ad}([\mathfrak{g}, \mathfrak{g}]) \oplus \mathbb{C} E \subset$ $\mathfrak{g l}([\mathfrak{g}, \mathfrak{g}])$, then condition (1.1) holds.

Theorem 1.3. Suppose that the (simple) linear Lie algebra $R([\mathfrak{g}, \mathfrak{g}])$ is classical. Condition (1.1) holds if and only if the linear algebra $\mathfrak{g} \subset \mathfrak{s l}(V)$ is reducible, and the complex linear Lie algebra $\mathfrak{g} \subset \mathfrak{g l}_{\mathbb{C}}\left(V_{\mathbb{R}}\right)$ coincides with one of the linear algebras $\mathfrak{s o}_{2 r-1} \oplus \mathbb{C} E$ $(r=2,3), \mathfrak{s l}_{3}, \mathfrak{g l}_{2}$, or $\mathfrak{s p}_{4}$. 
In 92 we introduce some notation we use in the paper, and prove some auxiliary results. In $\sqrt[3]{3}$ we prove Theorems 1.1 1.3. Finally, all the requisite tables are placed in 4 .

The author thanks Professor Ė. B. Vinberg for his supervision over many years, his constant support, and his valuable advice.

\section{$\S$ 2. Notation AND AUXiLiary RESUlts}

In this section all spaces (in particular, Lie algebras and their representations) are taken over the field $\mathbb{C}$ unless stipulated otherwise.

If $\mathfrak{g}_{i} \subset \mathfrak{s l}\left(V_{i}\right)(i=1, \ldots, m)$ are arbitrary linear Lie algebras, then the representation $R$ of the Lie algebra $\mathfrak{g}:=\mathfrak{g}_{1} \oplus \ldots \oplus \mathfrak{g}_{m}$ in the space $V:=V_{1} \otimes \ldots \otimes V_{m}$, which is the tensor product of the tautological representations $\mathfrak{g}_{i}: V_{i}(i=1, \ldots, m)$, is faithful, and we call the linear Lie algebra $R(\mathfrak{g}) \subset \mathfrak{s l}(V)$ isomorphic to the Lie algebra $\mathfrak{g}$ as an abstract algebra the tensor product of the linear Lie algebras $\mathfrak{g}_{i} \subset \mathfrak{s l}\left(V_{i}\right), i=1, \ldots, m$.

It is well known that any irreducible semisimple linear Lie algebra can be uniquely represented as the tensor product of irreducible simple linear Lie algebras called its simple components.

An ordered pair $(\Pi ; \alpha)$, where $\Pi$ is an indecomposable simple root system and $\alpha$ is its root with number $i$, is called a system with a marked root and denoted by $\Pi(\alpha)$ or by $\Pi(i)$. An isomorphism of two systems, each with a marked root, is defined to be an isomorphism of the corresponding indecomposable simple root systems taking the marked roots to one another. We say that two systems with a marked root are isomorphic if there exists an isomorphism between them, and we consider all systems with a marked root up to an isomorphism.

Let $\Omega$ denote the set of the following systems with a marked root:

1) $\Pi(1), \Pi \neq E_{8}$;

2) $A_{r}(2), r>2$

3) $A_{5}(3)$;

4) $B_{r}(r), r=3,4$

5) $C_{r}(2), r>2$

6) $D_{r}(r), r=5,6$.

For an indecomposable simple root system $\Pi$ we set $\partial \Pi:=\{\alpha \in \Pi: \Pi(\alpha) \in \Omega\} \subset \Pi$ and $\Pi:=\Pi \backslash(\partial \Pi)=\{\alpha \in \Pi: \Pi(\alpha) \notin \Omega\} \subset \Pi$. All the indecomposable simple root systems $\Pi$ for which $\partial \Pi \neq \Pi$ (or equivalently, $\Pi^{\circ} \neq \varnothing$ ) are listed in Table 1 , where the subsets $\partial \Pi \subset \Pi$ and $\stackrel{\circ}{\Pi} \subset \Pi$ are also indicated.

There is a natural bijection between the fundamental irreducible representations of an arbitrary simple Lie algebra and the roots of its (indecomposable) simple root system. This allows us to establish a bijection between all (up to an isomorphism) irreducible simple linear Lie algebras whose tautological representation is fundamental and all systems with a marked root. In what follows we shall identify each of the linear Lie algebras indicated above with the corresponding system with a marked root. Thus, the set $\Omega$ can be understood as a certain class of irreducible simple linear Lie algebras. We set $\widetilde{\Omega}$ to be the class of all simple linear Lie algebras of the set $\Omega$ that have rank greater than 1 .

Let $\mathfrak{g} \subset \mathfrak{g l}(V)$ be an irreducible reductive linear Lie algebra. Then the algebra $\mathfrak{g} \subset$ $\mathfrak{g l}(V)$ decomposes into the direct sum of its center and the derived subalgebra, which are contained in $\mathbb{C} E$ and $\mathfrak{s l}(V)$, respectively. Let $R$ denote the tautological representation $\mathfrak{g}: V$, and $\widetilde{R}$ the representation of the algebra $\mathfrak{g}$ equal to $R\left(R+R^{\prime}\right)$ if the representation $R$ is orthogonal (nonorthogonal, respectively).

Let $\mathfrak{g}_{i} \subset \mathfrak{s l}\left(V_{i}\right)(i=1, \ldots, m)$ be the simple components of the irreducible semisimple linear Lie algebra $[\mathfrak{g}, \mathfrak{g}] \subset \mathfrak{s l}(V)$. We set $r:=\operatorname{rk} \mathfrak{g}$ and $r_{i}:=\operatorname{rk} \mathfrak{g}_{i}(i=1, \ldots, m)$. 
Lemma 2.1. Suppose that $m>0, r_{1} \geq r-1$, and an s.s.g.p. of the representation $\widetilde{R}$ of the algebra $\mathfrak{g}$ is nontrivial. Then either $\mathfrak{g} \subset \mathfrak{g l}(V)$ is an adjoint simple linear Lie algebra, or the linear Lie algebra $\mathfrak{g}_{1} \subset \mathfrak{s l}\left(V_{1}\right)$ belongs to the class $\Omega$.

Proof. Consider an arbitrary number $i=1, \ldots, m$. Let $R_{i}$ denote the tautological representation $\mathfrak{g}_{i}: V_{i}$. We set $n_{i}:=\operatorname{dim} V_{i}$. Next, let $l_{i}\left(L_{i}\right)$ denote the index of the simple linear Lie algebra $\mathfrak{g}_{i}$ (the simple linear Lie algebra $\mathfrak{g}_{i}$ as a subalgebra of the linear Lie algebra $[\mathfrak{g}, \mathfrak{g}] \subset \mathfrak{s l}(V)$, respectively). Every time we talk about the index of a simple component $\mathfrak{g}_{i}$ of the irreducible semisimple linear Lie algebra $[\mathfrak{g}, \mathfrak{g}] \subset \mathfrak{s l}(V)$, we mean the number $L_{i}$.

Obviously, for any $i=1, \ldots, m$ the number $L_{i}$ is equal to the product of the number $l_{i}$ and all the numbers $n_{j}, j \in\{1, \ldots, m\} \backslash\{i\}$.

The following cases are possible.

Case 1. The algebra $\mathfrak{g}$ is simple, which is equivalent to $\mathfrak{g}_{1}=\mathfrak{g}$.

Case 2 . The center of the algebra $\mathfrak{g}$ is nontrivial, and the linear algebra $\mathfrak{g}_{1}$ is not locally transitive.

Case 3 . The linear algebra $\mathfrak{g}_{1}$ is locally transitive.

Case 4. The linear algebra $\mathfrak{g}$ is semisimple but it is not simple and has at least one classical simple component of index less than 1 .

Case 5. The linear algebra $\mathfrak{g}$ is semisimple but is not simple, and all its classical simple components have index at least 1.

Suppose that Case 2 holds. Then $m=1, r_{1}=r-1, \mathfrak{g}_{1}=[\mathfrak{g}, \mathfrak{g}], V_{1}=V$ and $\mathfrak{g}=\mathbb{C} E \oplus \mathfrak{g}_{1} \subset \mathfrak{g l}(V)$. Therefore, the representation $\mathfrak{g}: V$ is nonorthogonal. Since the linear algebra $\mathfrak{g}_{1}$ is not locally transitive, an s.s.g.p. of this representation is contained in the subalgebra $\mathfrak{g}_{1} \subset \mathfrak{g}$; the same can also be said about an s.s.g.p. of the representation $\widetilde{R}$.

Thus, in both Case 1 and Case 2 the representation $\left.\widetilde{R}\right|_{\mathfrak{g}_{1}}$ of the algebra $\mathfrak{g}_{1}$ has a nontrivial s.s.g.p. and coincides with the representation $R_{1}$ or $R_{1}+R_{1}^{\prime}$; furthermore, the first option is only possible in Case 1 under the condition that the representation $\mathfrak{g}: V$ is orthogonal.

All the simple linear Lie algebras with a nontrivial s.s.g.p. are precisely all the adjoint simple linear Lie algebras, as well as all the linear Lie algebras listed in $\underline{6}$, Tables 1 and 2] and [9, Table 0]. If, from among the tautological representations of these linear Lie algebras, we choose the orthogonal irreducible representations and direct sums of two mutually dual irreducible representations (taking into account that the adjoint representation of an arbitrary simple Lie algebra is irreducible), we obtain the required assertion in each of the Cases 1 and 2 .

We now consider Case 3 , By the results in [8], any locally transitive irreducible simple linear Lie algebra can be obtained from the linear algebras $A_{r}(1), A_{2 r}(2), C_{r}(1)(r \in \mathbb{N})$, or $D_{5}(5)$ by using the duality principle. Applying this to an irreducible semisimple linear Lie algebra does not affect its simple components, other than the linear algebra $A_{r}(1)$, and thus the lemma is proved in Case 3 .

In what follows we assume that either Case 4 or Case 5 holds, and the linear algebra $\mathfrak{g}_{1}$ does not belong to the class $\Omega$.

The following assertions hold:

1) $m=2, r_{1}=r-1, r_{2}=1$, and $\mathfrak{g} \subset \mathfrak{s l}(V)$;

2) if the linear Lie algebra $\mathfrak{g}_{1}$ is classical, then $\mathfrak{g}_{1}=\mathfrak{s o}_{3}$;

3) if $n_{1}<4$, then $\mathfrak{g}_{1}=\mathfrak{s o}_{3}$;

4) if the linear Lie algebra $\mathfrak{g}_{2}$ is classical, then $\mathfrak{g}_{2}=\mathfrak{s l}_{2}$ or $\mathfrak{g}_{2}=\mathfrak{s o}_{3}$; 
5) none of the simple components of the irreducible semisimple linear Lie algebra $\mathfrak{g}$ belongs to the class $\widetilde{\Omega}$.

If $\mathfrak{g}_{i}=\mathfrak{s o}_{3}$ for some $i=1,2$, then $L_{i} \geq l_{i}=1$, and if $\mathfrak{g}_{2}=\mathfrak{s l}_{2}$, then $l_{2}=1 / 4$ and $L_{2}=n_{1} / 4$. Therefore in Case 4 the irreducible semisimple linear algebra $\mathfrak{g}$ has two simple components $\mathfrak{g}_{1}=\mathfrak{s o}_{3}$ and $\mathfrak{g}_{2}=\mathfrak{s l}_{2}$. For such a linear algebra $\mathfrak{g}$ the representation $R$ is symplectic, its s.s.g.p. is one-dimensional, the representation $R^{\prime}$ is faithful, and as a consequence, an s.s.g.p. of the representation $\widetilde{R}=R+R^{\prime}$ is trivial, which leads us to a contradiction in Case 4

All the irreducible semisimple linear Lie algebras that are not simple, do not have classical simple components of index less than 1, but have a nontrivial s.s.g.p. are listed in [7, Tables 5 and 6]. In these tables every linear Lie algebra is represented as a tensor product of irreducible semisimple linear Lie algebras $\mathfrak{g}_{i}^{\prime} \subset \mathfrak{s l}\left(V_{i}^{\prime}\right), i=1, \ldots, m^{\prime}$ (in this order). For all the linear algebras in Table 6, and also for linear algebras nos. 4, 7, 8 in Table 5 , the linear algebra $\mathfrak{g}_{1}^{\prime}$ belongs to the class $\widetilde{\Omega}$. Furthermore, the linear algebras nos. 2, 3, 6 in Table 5 each have three simple components. Therefore in Case 5 the linear algebra $\mathfrak{g}$ coincides with one of the linear algebras no. 1 or 5 of Table 5 , that is, $m^{\prime}=2$, $\mathfrak{g}_{1}^{\prime}=\mathfrak{s o}_{n_{1}}$, and $n_{1}=n_{2}+2$. Furthermore, if $n_{1}=3$, then $n_{2}=1, \mathfrak{g}_{1}^{\prime}=\mathfrak{s o}_{3}, \mathfrak{g}_{2}^{\prime}=0$, $m=1$; if $n_{1}=4$, then $n_{2}=2, \mathfrak{g}_{1}^{\prime}=\mathfrak{s o}_{4} \cong \mathfrak{s l}_{2} \oplus \mathfrak{s l}_{2}$, and $\mathfrak{g}_{2}^{\prime}=\mathfrak{s l}_{2}$, whence $m=3$; but if $n_{1}>4$, then the linear algebra $\mathfrak{g}_{1}^{\prime}=\mathfrak{s o}_{n_{1}}$ belongs to the class $\widetilde{\Omega}$. Thus we have also arrived at a contradiction in Case [5]

The lemma is now completely proved.

Lemma 2.2. Suppose that $r=1$, and an s.s.g.p. of the representation $\widetilde{R}$ of the algebra $\mathfrak{g}$ is nontrivial. Then $\mathfrak{g} \subset \mathfrak{g l}(V)$ is an adjoint simple linear Lie algebra $\operatorname{ad}\left(\mathfrak{s l}_{2}\right)$.

Proof. We have $\operatorname{rk} \mathfrak{g}=r=1<\operatorname{dim} \mathfrak{g}$, since an s.s.g.p. of the faithful representation $\widetilde{R}$ of the algebra $\mathfrak{g}$ is nontrivial. Therefore, $m=1, r_{1}=1=r>r-1, \mathfrak{g} \cong \mathfrak{s l}_{2}, V_{1}=V$, $\mathfrak{g}_{1}=\mathfrak{g} \subset \mathfrak{s l}(V)$. Suppose that the linear algebra $\mathfrak{g} \subset \mathfrak{s l}(V)$ is not adjoint. By Lemma 2.1. the linear algebra $\mathfrak{g} \subset \mathfrak{s l}(V)$ belongs to the class $\Omega$ and, in view of the equation $r=1$, coincides with the linear algebra $A_{1}(1)=\mathfrak{s l}_{2}$. The tautological representation $R$ of the algebra $\mathfrak{g}=\mathfrak{s l}_{2}$ is symplectic, and an s.s.g.p. of its representation $\widetilde{R}=R+R^{\prime}=R+R$ is trivial, which contradicts the hypothesis.

Assertion 2.1. Suppose that $\Pi$ and $\Pi^{\prime}$ are indecomposable systems of simple roots such that $\Pi^{\prime} \subset \Pi$ and $\left|\Pi^{\prime}\right|=|\Pi|-1$, and let $\alpha \in \Pi^{\prime}$ be some root. If $\Pi(\alpha) \in \Omega$, then $\Pi^{\prime}(\alpha) \in \Omega$.

Proof. If $\left|\Pi^{\prime}\right|=1$, then $\Pi^{\prime}(\alpha)=A_{1}(1) \in \Omega$. In what follows we assume that $|\Pi|>2$.

By hypothesis, $\Pi^{\prime}=\Pi \backslash\{\beta\}$, where $\beta \in \Pi \backslash\{\alpha\}$ is the root of the indecomposable simple root system $\Pi$ corresponding to a hanging vertex of its (indecomposable) Dynkin diagram. All possible systems with a marked root $\Pi^{\prime}(\alpha)$ that can be obtained in this fashion for $\Pi(\alpha) \in \Omega$ and $|\Pi|>2$ are listed in Table 2 (where it is assumed throughout that $r>2$ ). It remains to use Table 1 .

Corollary 2.1. Suppose that $\Pi$ and $\Pi^{\prime}$ are indecomposable simple root systems such that $\Pi^{\prime} \subset \Pi$ and $\left|\Pi^{\prime}\right| \geqslant|\Pi|-1$. If for a root $\alpha \in \Pi^{\prime}$ we have $\Pi(\alpha) \in \Omega$, then $\Pi^{\prime}(\alpha) \in \Omega$.

The following two assertions are presented without proof because they are well known.

Assertion 2.2. An irreducible representation of an arbitrary complex reductive Lie algebra $\mathfrak{g}$ is faithful if and only if its weights with respect to a Cartan subalgebra $\mathfrak{t} \subset \mathfrak{g}$ linearly generate the space $\mathbf{t}^{*}$. 
Assertion 2.3. Suppose that $\mathbb{E}$ is a Euclidean space, $\Pi \subset \mathbb{E}$ an indecomposable simple root system, and $C \subset \mathbb{E}$ a Weyl chamber of it. Then for any subset $\Pi^{\prime} \subset \Pi$ different from $\Pi$, we have $\left\langle\Pi^{\prime}\right\rangle_{\mathbb{R}} \cap C=\{0\}$.

\section{$\S 3$. Proofs of THE RESUlts}

This section is devoted to proving Theorems 1.11 .3 .

We return to the notation and assumptions in $₫ 1$.

We begin with the proof of Theorem 1.1.

We fix a maximal commutative subalgebra $\mathfrak{t}_{\mathbb{R}}$ of the algebra $\mathfrak{g}_{\mathbb{R}}$ and a Cartan subalgebra $\mathfrak{t}:=\mathfrak{t}_{\mathbb{R}}(\mathbb{C})$ of the algebra $\mathfrak{g}$. As a result, a root system $\Delta \subset \mathfrak{t}^{*}$, the Weyl group $W \subset \mathrm{GL}\left(\mathfrak{t}^{*}\right)$, a system of positive roots $\Delta^{+} \subset \Delta$, and a simple root system $\Pi \subset \Delta^{+} \subset \Delta$ arise. Since the algebra $[\mathfrak{g}, \mathfrak{g}]$ is simple, the simple root system $\Pi \subset \mathfrak{t}^{*}$ is indecomposable. Let $\mathscr{P}$ denote the family of all indecomposable simple root systems $\Pi^{\prime} \subset \Pi \subset \mathfrak{t}^{*}$ of order $r-2$. In the simple root system $\Pi$, the subset of all roots that do not belong to any of the subsets $\Pi^{\prime}\left(\Pi^{\prime} \in \mathscr{P}\right)$ is denoted by $\partial_{r} \Pi$.

Let $\Lambda \subset \mathfrak{t}^{*}$ be the weight system of the representation $R$, and $\lambda \in \Lambda$ its highest weight with respect to the simple root system $\Pi \subset \Delta$. We set $\Pi_{\lambda}:=\{\alpha \in \Pi:\langle\lambda \mid \alpha\rangle \neq 0\} \subset \Pi$.

We have $\langle W \lambda\rangle=\langle\Lambda\rangle=\mathfrak{t}^{*},\langle\{\lambda\} \cup \Pi\rangle=\mathfrak{t}^{*}, r=\operatorname{dim} \mathfrak{t}^{*} \in\{|\Pi| ;|\Pi|+1\},|\Pi| \in\{r ; r-1\}$. In particular, $r-2<|\Pi|$.

Assertion 3.1. Suppose that $r>2$. Then the simple root system $\Pi$ coincides with the union of all its subsets $\Pi^{\prime} \in \mathscr{P}$.

Proof. We have $0<r-2<|\Pi|$. Therefore, in the (indecomposable) Dynkin diagram of the simple root system $\Pi$, for any vertex there exists an indecomposable subdiagram of order $r-2$ containing it.

Let $\Pi^{\prime} \in \mathscr{P}$ be some simple root system satisfying the condition

$$
(r>2) \Rightarrow\left(\Pi_{\lambda} \cap \Pi^{\prime} \neq \varnothing\right),
$$

and let $W^{\prime} \subset \mathrm{GL}\left(\mathfrak{t}^{*}\right)$ be its Weyl group.

We have $\left|\Pi^{\prime}\right|=r-2<|\Pi|$. By Assertion 2.3, the system $\left(\{\lambda\} \cup \Pi^{\prime}\right) \subset \mathfrak{t}^{*}$ consists of $r-1$ linearly independent linear functions taking purely imaginary values on the real form $\mathfrak{t}_{\mathbb{R}}$ of the space $t$. Consequently, the intersection of the kernels of these linear functions has the form $\mathbb{C} \xi \subset \mathfrak{t}, \xi \in \mathfrak{t}_{\mathbb{R}} \backslash\{0\}$. In the space $\mathfrak{t}^{*}$, the subspace of all linear functions vanishing on the vector $\xi \in \mathfrak{t}_{\mathbb{R}}$ can be identified with the space $\mathfrak{t}_{0}^{*}$, where $\mathfrak{t}_{0}:=\mathfrak{t} /(\mathbb{C} \xi)$. Clearly, $\operatorname{dim} \mathfrak{t}_{0}=r-1$ and $\mathfrak{t}_{0}^{*}=\left\langle\{\lambda\} \cup \Pi^{\prime}\right\rangle \subset \mathfrak{t}^{*}$.

We claim that

$$
\Pi^{\prime} \subset\left\langle W^{\prime} \lambda\right\rangle .
$$

For any root $\alpha \in \Pi_{\lambda} \cap \Pi^{\prime}$ we have

$$
\alpha=\frac{1}{\langle\lambda \mid \alpha\rangle}\left(\lambda-r_{\alpha} \lambda\right) \in\left\langle W^{\prime} \lambda\right\rangle \quad \text { and } \quad \alpha \in\left\langle W^{\prime} \lambda\right\rangle \cap\left\langle\Pi^{\prime}\right\rangle .
$$

If $r>2$, then $\Pi_{\lambda} \cap \Pi^{\prime} \neq \varnothing$ and $\left\langle W^{\prime} \lambda\right\rangle \cap\left\langle\Pi^{\prime}\right\rangle \neq 0$, which implies (3.2), since the $W^{\prime}$-submodule $\left\langle\Pi^{\prime}\right\rangle \subset \mathfrak{t}^{*}$ is simple. If, however, $r=2$, then $\left|\Pi^{\prime}\right|=r-2=0$ and $\Pi^{\prime}=\varnothing \subset\left\langle W^{\prime} \lambda\right\rangle$.

By (3.2), $\mathfrak{t}_{0}^{*}=\left\langle\{\lambda\} \cup \Pi^{\prime}\right\rangle=\left\langle W^{\prime} \lambda\right\rangle \subset \mathfrak{t}^{*}$.

Let $\Delta_{0}$ denote the root system $\left(\Delta \cap \mathfrak{t}_{0}^{*}\right) \subset \mathfrak{t}_{0}^{*}$, and $\Delta_{0}^{+}$the system of positive roots $\left(\Delta^{+} \cap \mathfrak{t}_{0}^{*}\right) \subset \Delta_{0}$. The latter corresponds to the simple root system $\Pi_{0} \subset \Delta_{0}$. We have $\Pi^{\prime} \subset \Pi \subset \Delta^{+}$and $\Pi^{\prime} \subset \mathfrak{t}_{0}^{*}$, whence $\Pi^{\prime} \subset \Delta_{0}^{+} \subset \Delta_{0}$. Furthermore, $\Pi \cap\left(\Delta^{+}+\Delta^{+}\right)=\varnothing$, $\Pi^{\prime} \cap\left(\Delta_{0}^{+}+\Delta_{0}^{+}\right)=\varnothing$, and therefore, $\Pi^{\prime} \subset \Pi_{0}$. 
In the algebra $\mathfrak{g}$ the centralizer $\mathfrak{g}^{\prime}$ of the element $\xi \in \mathfrak{t}_{\mathbb{R}}$ agrees with its compact real form $\mathfrak{g}_{\mathbb{R}}$ and contains the Cartan subalgebra $\mathfrak{t} \subset \mathfrak{g}$. Therefore, $\mathfrak{g}^{\prime}$ is a complex reductive algebra with compact real form $\mathfrak{g}_{\mathbb{R}}^{\prime}:=\mathfrak{g}^{\prime} \cap \mathfrak{g}_{\mathbb{R}}$, Cartan subalgebra $\mathfrak{t} \subset \mathfrak{g}$, and root system $\Delta_{0} \subset \mathfrak{t}^{*}$, while $\mathbb{C} \xi$ is a central ideal of the algebra $\mathfrak{g}^{\prime}$ compatible with its compact real form $\mathfrak{g}_{\mathbb{R}}^{\prime}$. In turn, $\mathfrak{g}_{0}:=\mathfrak{g}^{\prime} /(\mathbb{C} \xi)$ is a complex reductive Lie algebra with compact real form $\mathfrak{g}_{\mathbb{R}}^{\prime} /(\mathbb{R} \xi)$, Cartan subalgebra $\mathfrak{t}_{0}$, and root system $\Delta_{0} \subset \mathfrak{t}_{0}^{*}$; furthermore, $\operatorname{rk} \mathfrak{g}_{0}=\operatorname{dim} \mathfrak{t}_{0}=r-1$.

Let $V_{0} \subset V$ be the kernel of the operator $\xi \in \mathfrak{t}_{\mathbb{R}} \subset \mathfrak{g} \subset \mathfrak{s l}(V)$. The restriction of the tautological representation $\mathfrak{g}^{\prime}: V$ to the (obviously invariant) subspace $V_{0} \subset V$ naturally induces a representation $\mathfrak{g}_{0}: V_{0}$, since $(\mathbb{C} \xi) V_{0}=0$. Furthermore,

$$
\begin{gathered}
V_{0}=\left(V_{0} \cap V_{\mathbb{R}}\right) \oplus i\left(V_{0} \cap V_{\mathbb{R}}\right)=\left(V_{0} \cap V_{\mathbb{R}}\right)(\mathbb{C}), \\
\left(\mathfrak{g}_{\mathbb{R}}^{\prime} /(\mathbb{R} \xi)\right)\left(V_{0} \cap V_{\mathbb{R}}\right)=\mathfrak{g}_{\mathbb{R}}^{\prime}\left(V_{0} \cap V_{\mathbb{R}}\right) \subset\left(V_{0} \cap V_{\mathbb{R}}\right) .
\end{gathered}
$$

The algebra $\mathfrak{g}_{0}$ has an irreducible representation $R_{0}$ with highest weight $\lambda \in \mathfrak{t}_{0}^{*}$ with respect to the system of positive roots $\Delta_{0}^{+} \subset \Delta_{0}$. If $\pi: \mathfrak{g}^{\prime} \rightarrow \mathfrak{g}_{0}$ is the canonical epimorphism of algebras, then $R_{0} \circ \pi$ is an irreducible representation of the algebra $\mathfrak{g}^{\prime}$ with highest weight $\lambda \in \mathfrak{t}^{*}$ with respect to the system of positive roots $\Delta_{0}^{+} \subset \Delta_{0}$. Let $\widetilde{R}_{0}$ denote the representation of the algebra $\mathfrak{g}_{0}$ equal to $R_{0}\left(R_{0}+R_{0}^{\prime}\right)$ if the representation $R_{0}$ is orthogonal (nonorthogonal).

Proposition 3.1. The representation $R_{0}$ of the algebra $\mathfrak{g}_{0}$ is faithful.

Proof. Let $\Lambda_{0} \subset \mathfrak{t}_{0}^{*}$ be the weight system of the representation $R_{0}$ of the algebra $\mathfrak{g}_{0}$. We have $\Pi^{\prime} \subset \Delta_{0}, W^{\prime} \lambda \subset \Lambda_{0}, \mathfrak{t}_{0}^{*}=\left\langle W^{\prime} \lambda\right\rangle \subset\left\langle\Lambda_{0}\right\rangle \subset \mathfrak{t}_{0}^{*},\left\langle\Lambda_{0}\right\rangle=\mathfrak{t}_{0}^{*}$. It remains to apply Assertion 2.2 .

Thus, we can identify the algebra $\mathfrak{g}_{0}$ with the irreducible reductive linear algebra $R_{0}\left(\mathfrak{g}_{0}\right)$ and talk about simple components of its derived subalgebra - simple linear Lie algebras $\mathfrak{g}_{i} \subset \mathfrak{s l}\left(V_{i}\right)(i=1, \ldots, m)$ - and the corresponding indecomposable components $\Pi_{1}, \ldots, \Pi_{m}$ (in this order) of the simple root system $\Pi_{0} \subset \Delta_{0}$.

If $\mathfrak{g}_{0}$ is an adjoint linear algebra, then $\lambda \in \mathfrak{t}_{0}^{*}$ is the highest root of the root system $\Delta_{0} \subset \mathfrak{t}_{0}^{*}$, whence $\lambda \in \Delta_{0} \subset \Delta \subset \mathfrak{t}^{*}$.

Lemma 3.1. Suppose that $r=2$, and an s.s.g.p. of the representation $\widetilde{R}_{0}$ of the algebra $\mathfrak{g}_{0}$ is nontrivial. Then $\lambda \in \Delta$.

Proof. It suffices to apply Lemma 2.2 to the linear algebra $\mathfrak{g}_{0}$ of rank $r-1=1$.

Lemma 3.2. Suppose that $r>2, \lambda \notin \Delta$, and an s.s.g.p. of the representation $\widetilde{R}_{0}$ of the algebra $\mathfrak{g}_{0}$ is nontrivial. Then the subset $\left(\Pi_{\lambda} \cap \Pi^{\prime}\right) \subset \Pi$ includes a unique root $\alpha \in \Pi$. Furthermore,

1) $\langle\lambda \mid \alpha\rangle=1$ and $\Pi^{\prime}(\alpha) \in \Omega$;

2) if the simple root system $\Pi_{0}$ is indecomposable, then $\Pi_{0}(\alpha) \in \Omega$, and $\langle\lambda \mid \beta\rangle=0$ for every $\beta \in \Pi_{0} \backslash\{\alpha\}$.

Proof. The indecomposable simple root system $\Pi^{\prime} \subset \mathfrak{t}_{0}^{*}$ of order $r-2>0$ is contained in the simple root system $\Pi_{0} \subset \mathfrak{t}_{0}^{*}$, and therefore also in one of its indecomposable components $\Pi_{i}, i=1, \ldots, m$ (in particular, $m>0$ ). Without loss of generality, we assume that $\Pi^{\prime} \subset \Pi_{1}$. We have

$$
\begin{gathered}
\left|\Pi_{1}\right| \leqslant\left|\Pi_{0}\right| \leqslant \operatorname{dim} \mathfrak{t}_{0}^{*}=r-1=\left|\Pi^{\prime}\right|+1, \\
\operatorname{rk} \mathfrak{g}_{1}=\left|\Pi_{1}\right| \geqslant\left|\Pi^{\prime}\right|=r-2=\operatorname{rk} \mathfrak{g}_{0}-1 .
\end{gathered}
$$


Since $r>2$, the subset $\left(\Pi_{\lambda} \cap \Pi^{\prime}\right) \subset \Pi$ is not empty and includes some root $\alpha \in \Pi$. Applying Lemma 2.1 to the linear algebra $\mathfrak{g}_{0}$ and taking (3.5) into account we find that the linear algebra $\mathfrak{g}_{1} \subset \mathfrak{s l}\left(V_{1}\right)$ belongs to the class $\Omega$. This means that

1) $\langle\lambda \mid \alpha\rangle=1$;

2) $\langle\lambda \mid \beta\rangle=0$ for every $\beta \in \Pi_{1} \backslash\{\alpha\}$ (in particular, $\langle\lambda \mid \beta\rangle=0$ for every $\beta \in \Pi^{\prime} \backslash\{\alpha\}$ );

3) $\Pi_{1}(\alpha) \in \Omega$ (and $\Pi^{\prime}(\alpha) \in \Omega$ by relation (3.4) and Corollary 2.1).

Lemma 3.3. Suppose that an s.s.g.p. of the representation $\widetilde{R}_{0}$ of the algebra $\mathfrak{g}_{0}$ is trivial. Then there exists a vector $v \in V_{\mathbb{R}}$ such that $\xi \in\left(\mathfrak{g}_{\mathbb{R}}\right)_{v}$ and $\operatorname{rk}\left(\left(\mathfrak{g}_{\mathbb{R}}\right)_{v}\right)=1$.

Proof. Recall that $\lambda \in \mathfrak{t}_{0}^{*} \subset \mathfrak{t}^{*}$ is the highest weight of the representation $R$ of the algebra $\mathfrak{g}$ with respect to the simple root system $\Pi \subset \Delta$. By (3.3), the representation $\widetilde{R}_{0}$ of the algebra $\mathfrak{g}_{0}$ embeds into the representation $\mathfrak{g}_{0}: V_{0}$. Therefore, an s.s.g.p. of the latter is trivial. By using again relations (3.3) we obtain that an s.s.g.p. of the representation $\left(\mathfrak{g}_{\mathbb{R}}^{\prime} /(\mathbb{R} \xi)\right):\left(V_{0} \cap V_{\mathbb{R}}\right)$ is trivial. Consequently, there exists a vector $v \in V_{0} \cap V_{\mathbb{R}}$ such that $\mathfrak{g}_{v} \cap \mathfrak{g}_{\mathbb{R}}^{\prime}=\mathbb{R} \xi$. Thus, $\left(\left(\mathfrak{g}_{\mathbb{R}}\right)_{v}\right) \cap \mathfrak{g}^{\prime}=\mathfrak{g}_{v} \cap \mathfrak{g}_{\mathbb{R}}^{\prime}=\mathbb{R} \xi$, that is, the subalgebra $\left(\mathfrak{g}_{\mathbb{R}}\right)_{v} \subset \mathfrak{g}_{\mathbb{R}}$ contains $\mathbb{R} \xi$ as a maximal commutative subalgebra and therefore has rank 1.

Remark. The scheme of proof of Lemma 3.3 is adopted from [10, where a similar method is used to find points that have a closed orbit and stabilizer of rank 1 for complex reductive linear Lie groups.

Lemmas 3.13 .3 imply the following lemma.

Lemma 3.4. Suppose that $\lambda \notin \Delta$, and there is no vector $v$ in the space $V_{\mathbb{R}}$ such that $\xi \in\left(\mathfrak{g}_{\mathbb{R}}\right)_{v}$ and $\operatorname{rk}\left(\left(\mathfrak{g}_{\mathbb{R}}\right)_{v}\right)=1$. Then $r>2$ and $\Pi_{\lambda} \cap \Pi^{\prime}=\{\alpha\} \subset \Pi(\alpha \in \Pi)$. Furthermore,

1) $\langle\lambda \mid \alpha\rangle=1$ and $\alpha \in \partial \Pi^{\prime}$

2) if the simple root system $\Pi_{0}$ is indecomposable, then $\alpha \in \partial \Pi_{0}$, as well as $\langle\lambda \mid \beta\rangle=0$ for every $\beta \in \Pi_{0} \backslash\{\alpha\}$.

Until now we have assumed that the simple root system $\Pi^{\prime} \in \mathscr{P}$ satisfying (3.1) is fixed. However, in what follows we shall choose it each time depending on the concrete situation. In this case, Lemma 3.4 implies the following lemma.

Lemma 3.5. Suppose that $\lambda \notin \Delta$, and condition (1.1) does not hold. Let $\Pi^{\prime} \in \mathscr{P}$ be a simple root system satisfying (3.1). In the space $\mathfrak{t}^{*}$, let $\Pi_{0}$ denote the simple root system corresponding to the system of positive roots $\Delta^{+} \cap\left\langle\{\lambda\} \cup \Pi^{\prime}\right\rangle$. Then $r>2$ and $\Pi_{\lambda} \cap \Pi^{\prime}=\{\alpha\} \subset \Pi(\alpha \in \Pi)$. Furthermore,

1) $\langle\lambda \mid \alpha\rangle=1$ and $\alpha \in \partial \Pi^{\prime}$

2) if the simple root system $\Pi_{0}$ is indecomposable, then $\alpha \in \partial \Pi_{0}$, as well as $\langle\lambda \mid \beta\rangle=0$ for every $\beta \in \Pi_{0} \backslash\{\alpha\}$.

Lemma 3.6. Suppose that $\lambda \notin \Delta$, and condition (1.1) does not hold. Then $r>2$, and for any simple root system $\Pi^{\prime} \in \mathscr{P}$ we have

$$
\begin{gathered}
\left|\Pi_{\lambda} \cap \Pi^{\prime}\right| \leqslant 1, \quad \Pi_{\lambda} \cap \stackrel{\circ}{\Pi}^{\prime}=\varnothing, \\
\forall \alpha \in \Pi_{\lambda} \cap \Pi^{\prime} \quad\langle\lambda \mid \alpha\rangle=1 .
\end{gathered}
$$

Proof. Suppose that $r=2$. Then the simple root system $\Pi^{\prime}:=\varnothing \subset \Pi$ belongs to the family $\mathscr{P}$ and satisfies (3.1). Applying Lemma 3.5 to it, we find that $r>2$ and thus arrive at a contradiction.

Consequently, $r>2$. Let $\Pi^{\prime} \in \mathscr{P}$ be an arbitrary simple root system. If it satisfies (3.1), then relations (3.6) hold by Lemma 3.5. However, if (3.1) does not hold, then $\Pi_{\lambda} \cap \Pi^{\prime}=\varnothing$, which immediately implies (3.6). 
Lemma 3.7. Suppose that $\lambda \notin \Delta$, and condition (1.1) does not hold. Then $r>2$, $\Pi_{\lambda} \subset \partial_{r} \Pi$,

$$
\begin{gathered}
\forall \Pi^{\prime} \in \mathscr{P} \quad\left|\Pi_{\lambda} \cap \Pi^{\prime}\right| \leqslant 1, \\
\forall \alpha \in \Pi_{\lambda} \quad\langle\lambda \mid \alpha\rangle=1 .
\end{gathered}
$$

Proof. It follows from Lemma 3.6 that $r>2$ and $\Pi_{\lambda} \subset \partial_{r} \Pi$, and relation (3.7) holds. By applying Assertion 3.1 and Lemma 3.6 again, we now obtain (3.8).

We present a more convenient and fundamental interpretation of Lemma 3.7

Theorem 3.1. Suppose that $\lambda \notin \Delta$ and condition (1.1) does not hold. Then

1) $r>2$;

2) $\langle\lambda \mid \alpha\rangle=0$ for every $\alpha \in \Pi \backslash\left(\partial_{r} \Pi\right)$;

3) $\langle\lambda \mid \alpha\rangle \in\{0 ; 1\}$ for every $\alpha \in \partial_{r} \Pi$;

4) on the Dynkin diagram of the simple root system $\Pi$, there are vertices which correspond to any distinct roots $\alpha, \beta \in \Pi$ such that $\langle\lambda \mid \alpha\rangle=\langle\lambda \mid \beta\rangle=1$ and any path between these vertices contains at least $r-2$ edges.

Based on Table 1 all the cases where $r>2, \Pi$ is an indecomposable simple root system of order $r(r-1), \Pi^{\prime} \in \mathscr{P}$, and $\stackrel{\circ}{\Pi}^{\prime} \neq \varnothing$ are described, with the subset $\stackrel{\circ}{\Pi}^{\prime} \subset \Pi$ being indicated, and placed in Table 3 (in Table 5. respectively). Next, all the cases where $r>2, \Pi$ is an indecomposable simple root system of order $r(r-1)$, and $\partial_{r} \Pi \neq \Pi$ are given, with the subset $\partial_{r} \Pi \subset \Pi$ being indicated, and put in Table 4 (in Table 6) composed on the basis of Table 3 (Table 5, respectively).

Proposition 3.2. Suppose that $\lambda \in \Delta$. Then $\mathfrak{g}=[\mathfrak{g}, \mathfrak{g}]$, and the (simple) linear algebra $R(\mathfrak{g})$ is adjoint or coincides with one of the simple linear algebras $\varphi_{1}\left(B_{r}\right), \varphi_{2}\left(C_{r}\right)$, $\varphi_{1}\left(F_{4}\right)$, or $\varphi_{1}\left(G_{2}\right)$.

Proof. We have $\lambda \in \Delta \subset\langle\Pi\rangle \subset \mathfrak{t}^{*},\langle\Pi\rangle=\langle\{\lambda\} \cup \Pi\rangle=\mathfrak{t}^{*},|\Pi|=\operatorname{dim} \mathfrak{t}^{*}=r, \mathfrak{g}=[\mathfrak{g}, \mathfrak{g}]$. Furthermore, in the root system $\Delta$ with simple root system $\Pi$, the root $\lambda$ is dominant and therefore coincides with the highest root or (if there are roots of two different lengths) with the largest short root.

Proposition 3.3. Suppose that $\Pi=B_{r}, \lambda \notin \Delta,\left\langle\lambda \mid \alpha_{r}\right\rangle=0$, while condition (1.1) does not hold. Then $\left\langle\lambda \mid \alpha_{1}\right\rangle=\ldots=\left\langle\lambda \mid \alpha_{r-2}\right\rangle=0$.

Proof. Suppose that $\left\langle\lambda \mid \alpha_{i}\right\rangle \neq 0$ for some $i \in\{1, \ldots,[0] r-2\}$.

The simple root system $\Pi^{\prime}:=\left\{\alpha_{1}, \ldots, \alpha_{r-2}\right\} \subset \Pi$ belongs to the family $\mathscr{P}$ and satisfies (3.1). It is easy to see that $\left\langle\{\lambda\} \cup \Pi^{\prime}\right\rangle=\left\langle\alpha_{r}\right\rangle^{\perp}$. In the space $\mathfrak{t}^{*}$, let $\Pi_{0}$ denote the simple root system corresponding to the system of positive roots $\Delta^{+} \cap\left\langle\{\lambda\} \cup \Pi^{\prime}\right\rangle$. We have $\alpha:=\alpha_{r-1}+\alpha_{r} \in \Delta^{+}, h_{\alpha}=2 h_{\alpha_{r-1}}+h_{\alpha_{r}},\langle\lambda \mid \alpha\rangle=2\left\langle\lambda \mid \alpha_{r-1}\right\rangle+\left\langle\lambda \mid \alpha_{r}\right\rangle$, and $\Pi_{0}$ is an indecomposable simple root system of type $B_{r-1}$ including the simple roots $\alpha_{1}, \ldots, \alpha_{r-2}, \alpha$ in the standard order, whence $\partial \Pi_{0} \subset\left\{\alpha_{1}, \alpha\right\}$. By Lemma 3.5, $i=1$, $\left\langle\lambda \mid \alpha_{1}\right\rangle=1,0=\left\langle\lambda \mid \alpha_{2}\right\rangle=\ldots=\left\langle\lambda \mid \alpha_{r-2}\right\rangle=\langle\lambda \mid \alpha\rangle=2\left\langle\lambda \mid \alpha_{r-1}\right\rangle+\left\langle\lambda \mid \alpha_{r}\right\rangle$ and, as a consequence, $\left\langle\lambda \mid \alpha_{1}\right\rangle=1,0=\left\langle\lambda \mid \alpha_{2}\right\rangle=\ldots=\left\langle\lambda \mid \alpha_{r-2}\right\rangle=\left\langle\lambda \mid \alpha_{r-1}\right\rangle=\left\langle\lambda \mid \alpha_{r}\right\rangle, \lambda=\varphi_{1} \in \Delta$. We have obtained a contradiction.

Proposition 3.4. Suppose that $\Pi=C_{r}, \lambda \notin \Delta,\left\langle\lambda \mid \alpha_{r}\right\rangle=0$, while condition (1.1) does not hold. Then $\lambda=\varphi_{1}$ or $\left\langle\lambda \mid \alpha_{1}\right\rangle=\ldots=\left\langle\lambda \mid \alpha_{r-2}\right\rangle=0$.

Proof. Suppose that $\left\langle\lambda \mid \alpha_{i}\right\rangle \neq 0$ for some $i \in\{1, \ldots,[0] r-2\}$.

The simple root system $\Pi^{\prime}:=\left\{\alpha_{1}, \ldots, \alpha_{r-2}\right\} \subset \Pi$ belongs to the family $\mathscr{P}$ and satisfies (3.1). It is easy to see that $\left\langle\{\lambda\} \cup \Pi^{\prime}\right\rangle=\left\langle\alpha_{r}\right\rangle^{\perp}$. In the space $\mathfrak{t}^{*}$, let $\Pi_{0}$ denote the simple root system corresponding to the system of positive roots $\Delta^{+} \cap\left\langle\{\lambda\} \cup \Pi^{\prime}\right\rangle$. We have 
$\alpha:=2 \alpha_{r-1}+\alpha_{r} \in \Delta^{+}, h_{\alpha}=h_{\alpha_{r-1}}+h_{\alpha_{r}},\langle\lambda \mid \alpha\rangle=\left\langle\lambda \mid \alpha_{r-1}\right\rangle+\left\langle\lambda \mid \alpha_{r}\right\rangle$, and $\Pi_{0}$ is a system of indecomposable simple roots of type $C_{r-1}$ including the simple roots $\alpha_{1}, \ldots, \alpha_{r-2}, \alpha$ in the standard order, whence $\partial \Pi_{0}=\left\{\alpha_{1}, \alpha_{2}\right\}$. By Lemma 3.5, $i \in\{1,2\},\left\langle\lambda \mid \alpha_{j}\right\rangle=\delta_{i j}$ for every $j=1, \ldots, r-2$, and also $\langle\lambda \mid \alpha\rangle=0$. Therefore, $\left\langle\lambda \mid \alpha_{r-1}\right\rangle+\left\langle\lambda \mid \alpha_{r}\right\rangle=\langle\lambda \mid \alpha\rangle=0$, $\left\langle\lambda \mid \alpha_{r-1}\right\rangle=\left\langle\lambda \mid \alpha_{r}\right\rangle=0$, which implies that $\left\langle\lambda \mid \alpha_{j}\right\rangle=\delta_{i j}$ for any $j=1, \ldots, r$. We have thus shown that $\lambda=\varphi_{i} \in\left\{\varphi_{1}, \varphi_{2}\right\}$ and $\lambda=\varphi_{1}$, since $\varphi_{2} \in \Delta$.

Proposition 3.5. Suppose that $r>3, \Pi=D_{r}, \lambda \notin \Delta \cup\left\{\varphi_{1}\right\},\left\langle\lambda \mid \alpha_{r-1}\right\rangle=\left\langle\lambda \mid \alpha_{r}\right\rangle$, but condition (1.1) does not hold. Then $\left\langle\lambda \mid \alpha_{1}\right\rangle=\ldots=\left\langle\lambda \mid \alpha_{r-2}\right\rangle=0$.

Proof. Suppose that $\left\langle\lambda \mid \alpha_{i}\right\rangle \neq 0$ for some $i \in\{1, \ldots,[0] r-2\}$.

The simple root system $\Pi^{\prime}:=\left\{\alpha_{1}, \ldots, \alpha_{r-2}\right\} \subset \Pi$ belongs to the family $\mathscr{P}$ and satisfies (3.1), and $\left\langle\{\lambda\} \cup \Pi^{\prime}\right\rangle=\left\langle\alpha_{r-1}-\alpha_{r}\right\rangle^{\perp}$. In the space $\mathfrak{t}^{*}$, let $\Pi_{0}$ denote the simple root system corresponding to the system of positive roots $\Delta^{+} \cap\left\langle\{\lambda\} \cup \Pi^{\prime}\right\rangle$. For the root $\alpha:=$ $\alpha_{r-2}+\alpha_{r-1}+\alpha_{r} \in \Delta^{+}$we have $h_{\alpha}=h_{\alpha_{r-2}}+h_{\alpha_{r-1}}+h_{\alpha_{r}},\langle\lambda \mid \alpha\rangle=\left\langle\lambda \mid \alpha_{r-2}\right\rangle+\left\langle\lambda \mid \alpha_{r-1}\right\rangle+$ $\left\langle\lambda \mid \alpha_{r}\right\rangle$, and $\Pi_{0}$ is an indecomposable simple root system of type $D_{r-1}$ including the simple roots $\alpha_{1}, \ldots, \alpha_{r-2}, \alpha$ in the standard order, so that $\partial \Pi_{0} \subset\left\{\alpha_{1}, \alpha_{r-2}, \alpha\right\}$. By Lemma 3.5. $i \in\{1, r-2\},\left\langle\lambda \mid \alpha_{j}\right\rangle=\delta_{i j}$ for every $j=1, \ldots, r-2$, and also $\langle\lambda \mid \alpha\rangle=0$. Hence, $\left\langle\lambda \mid \alpha_{r-2}\right\rangle+\left\langle\lambda \mid \alpha_{r-1}\right\rangle+\left\langle\lambda \mid \alpha_{r}\right\rangle=\langle\lambda \mid \alpha\rangle=0$, and so $\left\langle\lambda \mid \alpha_{r-2}\right\rangle=\left\langle\lambda \mid \alpha_{r-1}\right\rangle=\left\langle\lambda \mid \alpha_{r}\right\rangle=0$, $i \neq r-2, i=1$, which implies that $\left\langle\lambda \mid \alpha_{j}\right\rangle=\delta_{1 j}$ for any $j=1, \ldots, r$. Therefore, $\lambda=\varphi_{1}$. We thus have a contradiction.

Corollary 3.1. Suppose that $\lambda \notin \Delta$, but condition (1.1) does not hold. Then

1) $r>2$

2) if $\Pi=B_{r}$, then $\lambda \in\left\{\varphi_{r-1}, \varphi_{r}, \varphi_{1}+\varphi_{r}, \varphi_{2}+\varphi_{r}, \varphi_{1}+\varphi_{2}+\varphi_{r}\right\}$;

3) if $r>3$ and $\Pi=B_{r}$, then $\lambda \in\left\{\varphi_{r-1}, \varphi_{r}, \varphi_{1}+\varphi_{r}, \varphi_{2}+\varphi_{r}\right\}$;

4) if $\Pi=C_{r}$, then $\lambda \in\left\{\varphi_{1}, \varphi_{r-1}, \varphi_{r}, \varphi_{1}+\varphi_{r}, \varphi_{2}+\varphi_{r}, \varphi_{1}+\varphi_{2}+\varphi_{r}\right\}$;

5) if $r>3$ and $\Pi=C_{r}$, then $\lambda \in\left\{\varphi_{1}, \varphi_{r-1}, \varphi_{r}, \varphi_{1}+\varphi_{r}, \varphi_{2}+\varphi_{r}\right\}$;

6) if $r>3$ and $\Pi=D_{r}$, then $\lambda \in\left\{\varphi_{1}, \varphi_{r-1}, \varphi_{r}, \varphi_{1}+\varphi_{r-1}, \varphi_{1}+\varphi_{r}, \varphi_{r-1}+\varphi_{r}\right\}$;

7) if $r>4$ and $\Pi=D_{r}$, then $\lambda \in\left\{\varphi_{1}, \varphi_{r-1}, \varphi_{r}, \varphi_{1}+\varphi_{r-1}, \varphi_{1}+\varphi_{r}\right\}$.

Proof. The proof follows from Theorem 3.1 and Propositions 3.33 .5

If we conduct a detailed analysis based on Theorem 3.1, Proposition 3.2, Corollary 3.1. and also Tables 4 and 6 we obtain the assertion of Theorem 1.1 .

We now turn to proving Theorems 1.2 and 1.3 .

Suppose that the (simple) linear Lie algebra $R([\mathfrak{g}, \mathfrak{g}])$ is adjoint or classical.

Let $\mathfrak{h}_{\mathbb{R}} \subset \mathfrak{g}_{\mathbb{R}}$ be an s.s.g.p. of the linear Lie algebra $\mathfrak{g}_{\mathbb{R}} \subset \mathfrak{s l}\left(V_{\mathbb{R}}\right)$, and $\mathfrak{t}_{[\mathfrak{g}, \mathfrak{g}]} \subset[\mathfrak{g}, \mathfrak{g}]$ a Cartan subalgebra of the algebra $[\mathfrak{g}, \mathfrak{g}]$.

The following assertion is obvious.

Assertion 3.2. If $\mathrm{rk} \mathfrak{h}_{\mathbb{R}}=1$, then condition (1.1) holds, while if $\mathrm{rk} \mathfrak{h}_{\mathbb{R}}>1$, condition (1.1) does not hold.

The following cases are possible.

Case 1. The linear Lie algebra $R([\mathfrak{g}, \mathfrak{g}])$ is an adjoint simple linear Lie algebra, the algebra $\mathfrak{g}$ has one-dimensional center, and the tautological representation $\mathfrak{g}: V$ coincides with $R+R^{\prime}$. The linear Lie algebra $R([\mathfrak{g}, \mathfrak{g}])$ is not locally transitive, which implies that $\mathfrak{t}_{[\mathfrak{g}, \mathfrak{g}]} \subset \mathfrak{g}$ is an s.s.g.p. of the representation $R$ of the algebra $\mathfrak{g}$. We have $\operatorname{dim} \mathfrak{t}_{[\mathfrak{g}, \mathfrak{g}]}=$ $\operatorname{rk}[\mathfrak{g}, \mathfrak{g}]=r-1 \geqslant 1$. Therefore, the root system of the simple Lie algebra $[\mathfrak{g}, \mathfrak{g}]$ with respect to its Cartan subalgebra $\mathfrak{t}_{[\mathfrak{g}, \mathfrak{g}]}$ contains some subset that linearly generates a hyperplane in $\mathfrak{t}_{[\mathfrak{g}, \mathfrak{g}]}^{*}$. Hence the representation $\left.R^{\prime}\right|_{\mathfrak{t}_{[\mathfrak{g}, \mathfrak{g}]}}$ of the algebra $\mathfrak{t}_{[\mathfrak{g}, \mathfrak{g}]}$ has a vector with onedimensional stationary subalgebra; the same can also be said about the tautological representation $\mathfrak{g}: V$. Thus, condition (1.1) holds. 
Case 2. The linear Lie algebra $R([\mathfrak{g}, \mathfrak{g}])$ coincides with the linear algebra $\mathfrak{s o}_{n}(\mathbb{C})$, where $n \in\{2 r-2,2 r-1\}$ and $n>4$, the algebra $\mathfrak{g}$ has a one-dimensional center, and the tautological representation $\mathfrak{g}: V$ coincides with $R+R^{\prime}$. Next, the linear Lie algebra $R([\mathfrak{g}, \mathfrak{g}])$ is not locally transitive. Hence an s.s.g.p. of the tautological representation $\mathfrak{g}: V$ is none other than an s.s.g.p. of the representation $\left.\left(R+R^{\prime}\right)\right|_{[\mathfrak{g}, \mathfrak{g}]}$ of the algebra $[\mathfrak{g}, \mathfrak{g}]$ which, in turn, is isomorphic to the Lie algebra $\mathfrak{s o}_{n-2}(\mathbb{C})$ of rank $r-2$. Thus, $\operatorname{rk} \mathfrak{h}_{\mathbb{R}}=r-2$. Therefore, $\operatorname{rk} \mathfrak{h}_{\mathbb{R}}=1$ for $n=5$, and $\operatorname{rk} \mathfrak{h}_{\mathbb{R}}>1$ otherwise.

Case 3. The tautological representation $\mathfrak{g}_{\mathbb{R}}: V_{\mathbb{R}}$ is obtained from the representation

$$
\operatorname{Mat}_{(r-1) \times(r-1)}(\mathbb{H}) \oplus \operatorname{Mat}_{1 \times 1}(\mathbb{H}): \operatorname{Mat}_{(r-1) \times 1}(\mathbb{H}), \quad(A+B): X \rightarrow A X-X B
$$

$(r>2)$ by a restriction to the subalgebra

$$
\left(\mathfrak{u}_{r-1}(\mathbb{H}) \oplus \mathbf{i} \mathbb{R} E\right) \subset\left(\operatorname{Mat}_{(r-1) \times(r-1)}(\mathbb{H}) \oplus \operatorname{Mat}_{1 \times 1}(\mathbb{H})\right),
$$

which is the direct sum of the subalgebras $\mathfrak{u}_{r-1}(\mathbb{H}) \subset \operatorname{Mat}_{(r-1) \times(r-1)}(\mathbb{H})$ and $\mathbf{i} \mathbb{R} E \subset$ $\operatorname{Mat}_{1 \times 1}(\mathbb{H})$. It is easy to see that $\mathfrak{h}_{\mathbb{R}} \cong \mathfrak{u}_{r-2}(\mathbb{H}) \oplus \mathbb{R}$. Hence, rk $\mathfrak{h}_{\mathbb{R}}=r-1>1$.

Case 4. The linear algebra $\mathfrak{g}_{\mathbb{R}} \subset \mathfrak{s l}\left(V_{\mathbb{R}}\right)$ is an adjoint simple compact linear Lie algebra. The subalgebra $\mathfrak{h}_{\mathbb{R}} \subset \mathfrak{g}_{\mathbb{R}}$ is a maximal commutative subalgebra of the algebra $\mathfrak{g}_{\mathbb{R}}$, whence, $\operatorname{rk} \mathfrak{h}_{\mathbb{R}}=\operatorname{rk} \mathfrak{g}_{\mathbb{R}}=r>1$.

Case 5. The linear algebra $\mathfrak{g}_{\mathbb{R}} \subset \mathfrak{s l}\left(V_{\mathbb{R}}\right)$ coincides with one of the linear algebras $\mathfrak{s o}_{2 r}(\mathbb{R})$ $(r>2), \mathfrak{s o}_{2 r+1}(\mathbb{R}), \mathfrak{s u}_{r+1}(\mathbb{C}), \mathfrak{u}_{r}(\mathbb{C}), \mathfrak{u}_{r}(\mathbb{H})$. The subalgebra $\mathfrak{h}_{\mathbb{R}} \subset \mathfrak{g}_{\mathbb{R}}$ is isomorphic to the algebra $\mathfrak{s o}_{2 r-1}(\mathbb{R}), \mathfrak{s o}_{2 r}(\mathbb{R}), \mathfrak{s u}_{r}(\mathbb{C}), \mathfrak{u}_{r-1}(\mathbb{C}), \mathfrak{u}_{r-1}(\mathbb{H})$, respectively, and has rank equal to $r$ for $\mathfrak{g}_{\mathbb{R}}=\mathfrak{s o}_{2 r+1}(\mathbb{R})$, and $r-1$ otherwise. Hence, rk $\mathfrak{h}_{\mathbb{R}}=1$ for $\mathfrak{g}_{\mathbb{R}}=\mathfrak{s u} 3(\mathbb{C})$, $\mathfrak{g}_{\mathbb{R}}=\mathfrak{u}_{2}(\mathbb{C})$, or $\mathfrak{g}_{\mathbb{R}}=\mathfrak{u}_{2}(\mathbb{H})$, and rk $\mathfrak{h}_{\mathbb{R}}>1$ otherwise.

Now, to prove Theorems 1.2 and 1.3 it suffices to use Assertion 3.2 .

\section{$\S$ 4. REFERENCE SECTION}

Table 1

\begin{tabular}{|r|l|l|l|}
\hline no. & \multicolumn{1}{|c|}{$\Pi$} & \multicolumn{1}{c|}{$\partial \Pi$} & \multicolumn{1}{c|}{$\Pi$} \\
\hline 1 & $A_{r}, r \geqslant 6$ & $1,2, r-1, r$ & $3, \ldots, r-2$ \\
\hline 2 & $B_{r}, r=3,4$ & $1, r$ & $2, \ldots, r-1$ \\
\hline 3 & $B_{r}, r \geqslant 5$ & 1 & $2, \ldots, r$ \\
\hline 4 & $C_{r}, r \geqslant 3$ & 1,2 & $3, \ldots, r$ \\
\hline 5 & $D_{r}, r=4,5,6$ & $1, r-1, r$ & $2, \ldots, r-2$ \\
\hline 6 & $D_{r}, r \geqslant 7$ & 1 & $2, \ldots, r$ \\
\hline 7 & $E_{6}$ & 1,5 & $2,3,4,6$ \\
\hline 8 & $E_{7}$ & 1 & $2, \ldots, 7$ \\
\hline 9 & $E_{8}$ & $\varnothing$ & $1, \ldots, 8$ \\
\hline 10 & $F_{4}$ & 1 & $2,3,4$ \\
\hline 11 & $G_{2}$ & 1 & 2 \\
\hline
\end{tabular}


Table 2

\begin{tabular}{|r|l|l|l|}
\hline no. & \multicolumn{1}{|c|}{$\Pi(\alpha)$} & \multicolumn{1}{|c|}{$i$} & $\left(\Pi \backslash\left\{\alpha_{i}\right\}\right)(\alpha)$ \\
\hline 1 & $A_{r}(1)$ & $r$ & $A_{r-1}(1)$ \\
\hline 2 & $A_{r}(2)$ & $r$ & $A_{r-1}(2)$ \\
\hline 3 & $A_{r}(2)$ & 1 & $A_{r-1}(1)$ \\
\hline 4 & $A_{5}(3)$ & 1 & $A_{4}(2)$ \\
\hline 5 & $B_{r}(1)$ & $r$ & $A_{r-1}(1)$ \\
\hline 6 & $B_{r}(r), r=3,4$ & 1 & $B_{r-1}(r-1)$ \\
\hline 7 & $C_{r}(1)$ & $r$ & $A_{r-1}(1)$ \\
\hline 8 & $C_{r}(2)$ & $r$ & $A_{r-1}(2)$ \\
\hline 9 & $C_{r}(2)$ & 1 & $C_{r-1}(1)$ \\
\hline 10 & $D_{r}(1)$ & $r$ & $A_{r-1}(1)$ \\
\hline 11 & $D_{r}(r), r=5,6$ & $r-1$ & $A_{r-1}(r-1)$ \\
\hline 12 & $D_{r}(r), r=5,6$ & 1 & $D_{r-1}(r-1)$ \\
\hline 13 & $E_{r}(1), r=6,7$ & $r$ & $A_{r-1}(1)$ \\
\hline 14 & $E_{r}(1), r=6,7$ & $r-1$ & $D_{r-1}(1)$ \\
\hline 15 & $F_{4}(1)$ & 4 & $C_{3}(1)$ \\
\hline
\end{tabular}

Table 3

\begin{tabular}{|r|l|l|l|l|}
\hline no. & \multicolumn{1}{|c|}{$\Pi$} & \multicolumn{2}{|c|}{$\Pi^{\prime}$} & \multicolumn{1}{|c|}{$\stackrel{\circ}{ }^{\prime}$} \\
\hline 1 & $A_{r}, r \geqslant 8$ & $3, \ldots, r$ & $A_{r-2}$ & $5, \ldots, r-2$ \\
\hline 2 & $A_{r}, r \geqslant 8$ & $2, \ldots, r-1$ & $A_{r-2}$ & $4, \ldots, r-3$ \\
\hline 3 & $A_{r}, r \geqslant 8$ & $1, \ldots, r-2$ & $A_{r-2}$ & $3, \ldots, r-4$ \\
\hline 4 & $B_{r}, r=5,6$ & $3, \ldots, r$ & $B_{r-2}$ & $4, \ldots, r-1$ \\
\hline 5 & $B_{r}, r \geqslant 7$ & $3, \ldots, r$ & $B_{r-2}$ & $4, \ldots, r$ \\
\hline 6 & $B_{r}, r \geqslant 8$ & $2, \ldots, r-1$ & $A_{r-2}$ & $4, \ldots, r-3$ \\
\hline 7 & $B_{r}, r \geqslant 8$ & $1, \ldots, r-2$ & $A_{r-2}$ & $3, \ldots, r-4$ \\
\hline 8 & $C_{r}, r \geqslant 5$ & $3, \ldots, r$ & $C_{r-2}$ & $5, \ldots, r$ \\
\hline 9 & $C_{r}, r \geqslant 8$ & $2, \ldots, r-1$ & $A_{r-2}$ & $4, \ldots, r-3$ \\
\hline 10 & $C_{r}, r \geqslant 8$ & $1, \ldots, r-2$ & $A_{r-2}$ & $3, \ldots, r-4$ \\
\hline 11 & $D_{r}, r=6,7,8$ & $3, \ldots, r$ & $D_{r-2}$ & $4, \ldots, r-2$ \\
\hline 12 & $D_{r}, r \geqslant 9$ & $3, \ldots, r$ & $D_{r-2}$ & $4, \ldots, r$ \\
\hline 13 & $D_{r}, r \geqslant 8$ & $2, \ldots, r-1$ & $A_{r-2}$ & $4, \ldots, r-3$ \\
\hline 14 & $D_{r}, r \geqslant 8$ & $2, \ldots, r-2, r$ & $A_{r-2}$ & $4, \ldots, r-3$ \\
\hline 15 & $D_{r}, r \geqslant 8$ & $1, \ldots, r-2$ & $A_{r-2}$ & $3, \ldots, r-4$ \\
\hline 16 & $E_{6}$ & $2,3,4,6$ & $D_{4}$ & 3 \\
\hline 17 & $E_{7}$ & $3, \ldots, 7$ & $D_{5}$ & 4,5 \\
\hline 18 & $E_{7}$ & $2, \ldots, 5,7$ & $D_{5}$ & 3,4 \\
\hline 19 & $E_{8}$ & $3, \ldots, 8$ & $E_{6}$ & $4,5,6,8$ \\
\hline 20 & $E_{8}$ & $2, \ldots, 6,8$ & $D_{6}$ & $3,4,5$ \\
\hline 21 & $E_{8}$ & $1, \ldots, 5,8$ & $A_{6}$ & 3,4 \\
\hline 22 & $E_{8}$ & $2, \ldots, 7$ & $A_{6}$ & 4,5 \\
\hline 23 & $E_{8}$ & $1, \ldots, 6$ & $A_{6}$ & 3,4 \\
\hline & & & \\
\hline
\end{tabular}


Table 4

\begin{tabular}{|r|l|l|}
\hline no. & \multicolumn{1}{|c|}{$\Pi$} & \multicolumn{1}{c|}{$\partial_{r} \Pi$} \\
\hline 1 & $A_{r}, r \geqslant 8$ & $1,2, r-1, r$ \\
\hline 2 & $B_{r}, r=5,6$ & $1,2,3, r$ \\
\hline 3 & $B_{7}$ & $1,2,3$ \\
\hline 4 & $B_{r}, r \geqslant 8$ & 1,2 \\
\hline 5 & $C_{r}, r=5,6,7$ & $1,2,3,4$ \\
\hline 6 & $C_{r}, r \geqslant 8$ & 1,2 \\
\hline 7 & $D_{r}, r=6,7$ & $1,2,3, r-1, r$ \\
\hline 8 & $D_{8}$ & $1,2,7,8$ \\
\hline 9 & $D_{r}, r \geqslant 9$ & 1,2 \\
\hline 10 & $E_{6}$ & $1,2,4,5,6$ \\
\hline 11 & $E_{7}$ & $1,2,6,7$ \\
\hline 12 & $E_{8}$ & $1,2,7$ \\
\hline
\end{tabular}

Table 5

\begin{tabular}{|r|l|l|l|l|}
\hline no. & \multicolumn{1}{|c|}{$\Pi$} & \multicolumn{2}{|c|}{$\Pi^{\prime}$} & \multicolumn{1}{c|}{$\stackrel{\circ}{ }^{\prime}$} \\
\hline 1 & $A_{r-1}, r \geqslant 8$ & $2, \ldots, r-1$ & $A_{r-2}$ & $4, \ldots, r-3$ \\
\hline 2 & $A_{r-1}, r \geqslant 8$ & $1, \ldots, r-2$ & $A_{r-2}$ & $3, \ldots, r-4$ \\
\hline 3 & $B_{r-1}, r=5,6$ & $2, \ldots, r-1$ & $B_{r-2}$ & $3, \ldots, r-2$ \\
\hline 4 & $B_{r-1}, r \geqslant 7$ & $2, \ldots, r-1$ & $B_{r-2}$ & $3, \ldots, r-1$ \\
\hline 5 & $B_{r-1}, r \geqslant 8$ & $1, \ldots, r-2$ & $A_{r-2}$ & $3, \ldots, r-4$ \\
\hline 6 & $C_{r-1}, r \geqslant 5$ & $2, \ldots, r-1$ & $C_{r-2}$ & $4, \ldots, r-1$ \\
\hline 7 & $C_{r-1}, r \geqslant 8$ & $1, \ldots, r-2$ & $A_{r-2}$ & $3, \ldots, r-4$ \\
\hline 8 & $D_{r-1}, r=6,7,8$ & $2, \ldots, r-1$ & $D_{r-2}$ & $3, \ldots, r-3$ \\
\hline 9 & $D_{r-1}, r \geqslant 9$ & $2, \ldots, r-1$ & $D_{r-2}$ & $3, \ldots, r-1$ \\
\hline 10 & $D_{r-1}, r \geqslant 8$ & $1, \ldots, r-2$ & $A_{r-2}$ & $3, \ldots, r-4$ \\
\hline 11 & $D_{r-1}, r \geqslant 8$ & $1, \ldots, r-3, r-1$ & $A_{r-2}$ & $3, \ldots, r-4$ \\
\hline 12 & $E_{6}$ & $2, \ldots, 6$ & $D_{5}$ & 3,4 \\
\hline 13 & $E_{6}$ & $1,2,3,4,6$ & $D_{5}$ & 2,3 \\
\hline 14 & $E_{7}$ & $2, \ldots, 7$ & $E_{6}$ & $3,4,5,7$ \\
\hline 15 & $E_{7}$ & $1, \ldots, 5,7$ & $D_{6}$ & $2,3,4$ \\
\hline 16 & $E_{7}$ & $1, \ldots, 6$ & $A_{6}$ & 3,4 \\
\hline 17 & $E_{8}$ & $2, \ldots, 8$ & $E_{7}$ & $3, \ldots, 8$ \\
\hline 18 & $E_{8}$ & $1, \ldots, 6,8$ & $D_{7}$ & $2, \ldots, 6,8$ \\
\hline 19 & $E_{8}$ & $1, \ldots, 7$ & $A_{7}$ & $3,4,5$ \\
\hline 20 & $F_{4}$ & $2,3,4$ & $B_{3}$ & 3 \\
\hline 21 & $F_{4}$ & $1,2,3$ & $C_{3}$ & 3 \\
\hline & & & \\
\hline
\end{tabular}


Table 6

\begin{tabular}{|r|l|l|}
\hline no. & \multicolumn{1}{|c|}{$\Pi$} & \multicolumn{1}{c|}{$\partial_{r} \Pi$} \\
\hline 1 & $A_{r-1}, r \geqslant 8$ & $1,2, r-2, r-1$ \\
\hline 2 & $B_{r-1}, r=5,6$ & $1,2, r-1$ \\
\hline 3 & $B_{r-1}, r \geqslant 7$ & 1,2 \\
\hline 4 & $C_{r-1}, r=5,6,7$ & $1,2,3$ \\
\hline 5 & $C_{r-1}, r \geqslant 8$ & 1,2 \\
\hline 6 & $D_{r-1}, r=6,7,8$ & $1,2, r-2, r-1$ \\
\hline 7 & $D_{r-1}, r \geqslant 9$ & 1,2 \\
\hline 8 & $E_{6}$ & $1,5,6$ \\
\hline 9 & $E_{7}$ & 1,6 \\
\hline 10 & $E_{8}$ & 1 \\
\hline 11 & $F_{4}$ & $1,2,4$ \\
\hline
\end{tabular}

\section{REFERENCES}

[1] O. G. Styrt, On the orbit space of a compact linear Lie group with a commutative connected component, Trudy Moskov. Mat. Ob-va 70 (2009), 235-287; English transl., Trans. Moscow Math. Soc. 2009, 171-206 MR2573640 (2011b:22019)

[2] O. G. Styrt, On the orbit space of a three-dimensional compact linear Lie group, Izv. Ross. Akad. Nauk Ser. Mat. 75 (2011), no. 4, 165-188; English transl., Izv. Math. 75 (2011), no. 4, 815-836. MR2866189(2012m:22024)

[3] O. G. Styrt, On the orbit space of a three-dimensional simple compact linear Lie group, Vestnik Moskov. Univ. Ser. I Mat. Mekh. 2010, no. 6, 51-52; English transl., Moscow Univ. Math. Bull. 65 (2010), no. 6, 255-256. MR2814991 (2012d:22032)

[4] G. E. Bredon, Introduction to compact transformation groups, Pure and Applied Mathematics, vol. 46, Academic Press, New York, London, 1972. MR.0413144 (54:1265)

[5] É. B. Vinberg and A. L. Onishchik, Seminar on Lie groups and algebraic groups, 2nd ed., URSS, Moscow, 1995; English transl. of first ed., A. L. Onishchik and E. B. Vinberg, Lie groups and algebraic groups, Springer Ser. in Soviet Math., Springer-Verlag, Berlin, 1990. MR1403378 (97d:22001)

[6] A. G. Élashvili, Canonical form and stationary subalgebras of points of general position for simple linear Lie groups, Funkts. Anal. Prilozh. 6 (1972), no. 1, 51-62; English transl., Funct. Anal. Appl. 6 (1972), 44-53. MR0304554 (46:3689)

[7] A. G. Élashvili, Stationary subalgebras of points of the common state for irreducible linear Lie groups, Funkts. Anal. Prilozh. 6 (1972), no. 2, 65-78; English transl., Funct. Anal. Appl. 6 (1972), 139-148. MR0304555(46:3690)

[8] G. B. Shpiz, Classification of irreducible locally transitive linear Lie groups, Geometric methods in problems of algebra and analysis, Yaroslavl' State Univ., Yaroslavl', 1978, 152-160. (Russian) MR.575878 (82j:22007)

[9] A. M. Popov, Finite stationary subgroups in general position of simple linear Lie groups, Trudy Moskov. Mat. Ob-va 48 (1985), 7-59. (Russian) MR830410 (87i:22021)

[10] V. G. Kac, V. L. Popov, and E. B. Vinberg, Sur les groupes linéaires algébriques dont l'algèbre des invariants est libre, C. R. Acad. Sci. Paris, 283 (1976), no. 12. 875-878. MR0419468 (54:7489)

Moscow State University

E-mail address: oleg_styrt@mail.ru

Translated by E. KHUKHRO

Originally published in Russian 